\title{
Enhancing coherence in molecular spin qubits via atomic clock transitions
}

Muhandis Shiddiq, ${ }^{1, *}$ Dorsa Komijani, ${ }^{1, *}$ Yan Duan, ${ }^{2}$ Alejandro Gaita-Arino, ${ }^{2}$ Eugenio Coronado, ${ }^{2}$ Stephen Hill ${ }^{1}$

${ }^{1}$ National High Magnetic Field Laboratory and Department of Physics, Florida State University, Tallahassee, Florida 32310 USA ${ }^{2}$ Instituto de Ciencia Molecular (ICMol), Universidad de Valencia,

C/ Catedrático José Beltrán 2, 46980 Paterna, Spain

${ }^{*}$ These authors contributed equally to this work

Quantum Computing is an emerging area within the information sciences revolving around the concept of quantum bits (or qubits). A major obstacle is the extreme fragility of these qubits due to interactions with their environment that destroy their "quantumness". This phenomenon, known as decoherence, is of immense fundamental interest. ${ }^{1,2}$ There are many competing candidates for qubits, including superconducting circuits, ${ }^{3}$ quantum optical cavities, ${ }^{4}$ ultracold atoms ${ }^{5}$ and spin qubits, ${ }^{6-8}$ and each one has its strengths and weaknesses. When dealing with spin qubits, the strongest source of decoherence is the magnetic dipolar interaction. ${ }^{9}$ To minimize it, spins are typically diluted in a diamagnetic matrix. For example, this dilution can be taken to the extreme of a single phosphorus atom in silicon, ${ }^{6}$ while in molecular matrices a typical ratio is one magnetic molecule per $10,000 .{ }^{10}$ However, there is a fundamental contradiction between reducing decoherence by dilution, and allowing quantum operations via the interaction between spin qubits. To solve this apparent paradox, the design and engineering of quantum hardware can benefit from a "bottom-up" approach whereby the electronic structure of magnetic molecules is chemically tailored to give the desired physical behavior. Here we present a very effective way of eliminating decoherence in solid-state molecular spin qubits without resorting to extreme dilution. It is based on the design of molecular structures with crystal field ground states possessing large tunneling gaps that give rise to optimal operating points, or atomic clock transitions, at which the quantum spin dynamics become protected against dipolar decoherence. This approach is illustrated with a holmium molecular nanomagnet in which long coherence times (up to $8.4 \mu \mathrm{s}$ at $5 \mathrm{~K}$ ) can be obtained at unusually high concentrations. This finding opens new avenues for quantum computing based on molecular spin qubits.

One of the proposed approaches to obtaining spin qubits is that of using magnetic molecules. ${ }^{8-16}$ Up to now, coherence has been optimized through dilution and deuteration to minimize dipolar and hyperfine interactions, respectively. ${ }^{10,16}$ A class of molecules in which these two sources of decoherence can be

34 minimized by alternative means are the so-called polyoxometalates. In the past, these metal-oxide 35 clusters have been used as model systems in molecular magnetism due to their ability to host magnetic 36 ions in chemically tailored environments of high symmetry and rigidity. ${ }^{17}$ Currently, these molecules are 37 seen as potential building blocks in quantum computing architectures. ${ }^{18-22}$ 
In the present study we chose the $\left[\mathrm{Ho}\left(\mathrm{W}_{5} \mathrm{O}_{18}\right)_{2}\right]^{9-}$ complex (abbreviated $\mathrm{HoW}$ 10) which has been subject to extensive structural, magnetic and spectroscopic characterizations that raised the possibility of observing coherent spin dynamics. ${ }^{23,24} \mathrm{HoW}_{10}$ is formed by two molecular tungsten oxide moieties encapsulating a $\mathrm{Ho}^{+3}$ ion (Fig. 1). The geometry around $\mathrm{Ho}^{+3}$ exhibits a slightly distorted square-antiprismatic environment, which can be approximated by a $D_{4 d}$ "pseudo-axial" symmetry. This results in a splitting of the $J=8$ ground state spin-orbit manifold according to its $m_{J}$ quantum numbers. Quantitatively this splitting can be described in terms of a crystal-field (CF) Hamiltonian (double summation in eq. 1) which, for $D_{4 d}$ symmetry, contains the axial CF terms $B_{2}^{0} \hat{O}_{2}^{0}, B_{4}^{0} \hat{O}_{4}^{0}$ and $B_{6}^{0} \hat{O}_{6}^{0}$ (see Methods for definition and discussion of terms in eq. 1$)^{24}$

$$
\hat{H}=\sum_{k=2,4,6} \sum_{q=0}^{k} B_{k}^{q} \hat{O}_{k}^{q}+\hat{J} \cdot \overrightarrow{\mathrm{A}} \cdot \hat{I}+\mu_{B} \overrightarrow{B_{0}} \cdot \vec{g} \cdot \hat{J}-\mu_{N} g_{N} \vec{B}_{0} \cdot \hat{I}
$$

This results in an isolated $m_{J}= \pm 4$ ground doublet, separated from the first excited states $\left(m_{J}= \pm 5\right)$ by $\sim 20 \mathrm{~cm}^{-1}$. This picture provides a reasonable description of the magnetic properties of this molecule. ${ }^{23}$ However, minor deviations from $D_{4 d}$ symmetry that are present in the crystal make operative the tetragonal $B_{4}^{4} \widehat{O}_{4}^{4} \mathrm{CF}$ interaction. Interestingly, the match between the ( \pm integer) values of the ground state spin projections, $m_{J}= \pm 4$, with the tetragonal (i.e., $q=4$ ) order of the main symmetry axis of the molecule, results in the $B_{4}^{4} \hat{O}_{4}^{4}\left[\hat{O}_{4}^{4}=\frac{1}{2}\left(\hat{J}_{+}^{4}+\hat{J}_{-}^{4}\right)\right]$ interaction generating an unusually large quantum tunneling gap, $\Delta \sim 9.18 \mathrm{GHz}\left(\sim 0.3 \mathrm{~cm}^{-1}\right){ }^{24}$ This gap is a crucial factor for the coherence of electron spin dynamics in molecular spin qubits, and is the main subject of the present study.

The standard approach for probing coherent spin dynamics involves the use of "electron spin echoes" in pulsed Electron Paramagnetic Resonance (EPR). The HoW 10 system is attractive in this regard because its predicted tunneling gap $(\sim 9.18 \mathrm{GHz}$, Fig. 1$)$ is close to the $\mathrm{X}$-band frequency associated with the most sophisticated EPR spectrometers. While the magnitude of the gap is set by $B_{4}^{4}$, interesting details of the EPR spectra are determined also by the hyperfine interaction between the Ho electron and nuclear spins (second term in eq. 1). Holmium occurs naturally in only one stable isotope $\left({ }^{165} \mathrm{Ho}\right)$ with a nuclear spin of $I=7 / 2$. A strong hyperfine coupling $\left(\mathrm{A}_{/ /}=830 \pm 10 \mathrm{MHz}\right)$ results in the observation of eight $(2 I+1)$ wellresolved transitions via continuous-wave (CW) high-frequency EPR measurements. ${ }^{24}$ The energy level scheme that arises from the combination of CF and hyperfine coupling, together with the Zeeman interaction $\left(3^{\text {rd }}\right.$ and $4^{\text {th }}$ terms in eq. 1 ), gives rise to a series of avoided level crossings between $m_{J}= \pm 4$ states (with the same $m_{l}$ ), resulting in multiple gaps in the energy diagram near zero-field (Fig. 1a). concentrations ranging from $x=0.25$ to $x=0.001$, i.e., up to three orders of magnitude away from the usual high-dilution limit, ${ }^{10}$ allowing a study of the effects of dilution on electron dipolar spin-spin 
decoherence. Fig. 2a displays electron-spin-echo- (ESE-) detected EPR spectra recorded at $5 \mathrm{~K}$ for a dilute $(x=0.001)$ sample at frequencies from 9.1 to $9.8 \mathrm{GHz}$, with $\theta=29^{\circ}\left(\theta\right.$ is the angle between $B_{0}$ and the $z$-axis of the crystal); ESE signals were generated using a two-pulse Hahn-echo sequence (see Methods). ${ }^{25}$ Four broad peaks of equal intensity are observed at the two lowest frequencies (9.11 and $9.18 \mathrm{GHz}$ ), which were selected to be close to the gap minima in Fig. 1b. With increasing frequency, these peaks split and move symmetrically apart, as expected on the basis of predictions in Fig. 1b. For the most part, the data lie on the simulated curves, with the obvious exception of the two lowest frequencies and some lower field $(<60 \mathrm{mT})$ data points. The simulations are based on previously determined Hamiltonian parameters, ${ }^{24}$ and the spectra are plotted against the re-scaled longitudinal applied field, $B_{0 z}\left(=B_{0} \cos \theta\right)$, to facilitate comparisons between different samples (see Methods).

Two-pulse ESE measurements were separately utilized to determine $5 \mathrm{~K}$ transverse relaxation times, $T_{2}$, at selected points within the spectrum for the $x=0.001$ concentration. The longest $T_{2}$ 's are found in the vicinity of the gap minima for the smallest crystals (see Figs. $2 b, 3$ and Methods), with values ranging from 5.2 to $8.4 \mu \mathrm{s}$, whereas the values are substantially shorter away from the minima. In fact, the $T_{2}$ values exhibit sharp divergences right at $B_{\min }$ (Fig. 3). The key to understanding this behavior is the quadratic field dependence of the EPR transition frequencies close to the gap minima (see Methods),

$$
f=\Delta+\frac{\gamma_{z}^{2}}{2 \Delta}\left(B_{0 z}-B_{\min }\right)^{2}
$$

such that the derivative $d f / d B_{0 z} \propto\left(B_{0 z}-B_{\min }\right) \rightarrow 0$ as $B_{0 z} \rightarrow B_{\min }$. Although not explicitly included in eq. 1, nearly all sources of dipolar decoherence (due, e.g., to dynamics associated with the nuclear bath and collective electron spin excitations, or magnons) can be approximated as a time-dependent magnetic noise, $\delta B_{0}(t)$, acting on the central spin qubit (the spin being measured) via the Zeeman interaction, i.e., processes that flip nearby spins cause variations in the local field, $\delta B_{0}$, at the position of the central spin, thereby altering its frequency/phase. Many of these processes involve indirect pairwise spin flip-flops (spin diffusion) that are extremely hard to mitigate, and persist to very low temperatures. The extreme axial anisotropy of $\mathrm{HoW}_{10}$ results in an insensitivity to the perpendicular applied field component, $B_{0 \perp}$ (see Methods). Meanwhile, sensitivity to $\delta B_{0 z}(\mathrm{t})$ vanishes (to first order) as $B_{0 z} \rightarrow B_{\min }$ and $d f / d B_{0 z} \rightarrow 0$, resulting in a vanishing contribution to the dipolar decoherence. This is the concept behind so-called 'atomic clock transitions'. Named after atomic clocks, these transitions are protected against environmental noise sources according to the principle described here (i.e., $d f / d B_{0}=0$ ), resulting in a clock frequency that exhibits exceptional phase stability. ${ }^{26,27}$ Indeed, one expects the dephasing time, $T_{2}$, to scale as $\left(B_{0 z}-B_{\min }\right)^{-n}\left(n>0\right.$, see Extended Data Fig. 1), ${ }^{28,29}$ thus explaining the observed divergences

107 at the clock-transitions (CTs). For comparison, $\mathrm{T}_{2}$ measurements are displayed in the right panel of Fig. 3 108 for several 'normal' EPR transitions, i.e., $m_{J}=-4$ to +4 transitions away from the gap minima, where the 
frequency dependence approaches the linear regime and $d f / d B_{0 z} \rightarrow \gamma_{z}=139.9 \mathrm{GHz} / \mathrm{T}$ (Fig. 1). Although

$110 \mathrm{~T}_{2}$ is moderately peaked at the centers of these resonances, the sharp divergences seen at the CTs are 111 clearly absent (see Methods for further discussion).

113 ESE-detected measurements for an $x=0.01$ sample reveal essentially identical divergences in $T_{2}$ at the 114 CTs to those seen in Fig. 3, with maximum values ranging from 4 to $8 \mu$ s (see Extended Data Figs. 1 and

115 2). However, $T_{2}$ values associated with 'normal' EPR transitions well away from the CTs are much shorter 116 ( $100 \mathrm{~ns}$, not shown). Because the collection of ESE spectra requires the detection of an echo, the 117 observation of these 'normal' EPR transitions is challenging for $x \geq 0.01$. These findings are consistent 118 with the idea that dipolar 'noise' increases with increasing Ho concentration, resulting in shorter $\mathrm{T}_{2}$ 's for 119 the 'normal' EPR transitions, yet an apparent insensitivity to the Ho concentration at the CTs.

Fig. 4 displays $5 \mathrm{~K}$ ESE-detected spectra for a concentrated $x=0.1$ sample which are in stark contrast to

122 those in Fig. 2: narrow resonances are observed at the CTs that do not shift at all with frequency, i.e., the 123 data do not follow the simulations even though CW measurements indicate no measurable variation in 124 the spin Hamiltonian parameters with Ho concentration. ${ }^{24}$ The total suppression of 'normal' EPR 125 transitions is attributed to a further reduction of $\mathrm{T}_{2}$ upon increasing the Ho concentration, to the extent that 126 an echo can no longer be detected. Nevertheless, the $T_{2}$ values at the CTs remain long $(\sim 0.7 \mu \mathrm{s})$, 127 resulting in the narrow ESE-detected resonances. Indeed, because the echo intensity is $T_{2}$-weighted, the 128 resonance lineshape is a direct manifestation of the field dependence of $\mathrm{T}_{2}$ at $B_{\min }$. Analysis of CW EPR 129 spectra suggests that the main contribution to the linewidth is a Gaussian distribution in the $B_{4}^{4}$ parameter $130\left(\sigma_{B 44}=0.63 \mathrm{MHz}\right)$. This causes significant vertical broadening of the tunneling gap, $\Delta$, and EPR transition 131 frequencies, as illustrated in Fig. $1 \mathrm{~b}$, which includes contours at the $\pm \sigma_{\Delta}$ and $\pm 2 \sigma_{\Delta}$ levels $\left(\sigma_{\Delta}=123 \mathrm{MHz}\right.$ is 132 the standard deviation in $\Delta$-see Methods). These simulations indicate measurable intensity at the CTs 133 up to at least $9.4 \mathrm{GHz}$. However, the $B_{4}^{4}$ distribution does not shift the CTs appreciably to lower or higher 134 fields, i.e., all molecules in the distribution have their CTs at essentially the same $B_{\min }$ values. This 135 explains the observation of narrow CT peaks spanning a wide frequency range in the $x=0.1$ sample 136 (Fig. 4); similar behavior is also discernible at other concentrations (see Extended Data Fig. 3).

138 After magnetic 'noise', several other sources of decoherence remain. First and foremost, the CTs do not 139 protect against direct flip-flop processes that involve the central spin qubit. ${ }^{28,29}$ These energy-conserving 140 events involve coupling to other spins via the off-diagonal component of the dipolar interaction $\left(\hat{S}_{1}^{+} \hat{S}_{2}^{-}+\right.$ $141 \hat{S}_{1}^{-} \hat{S}_{2}^{+}$). The inhomogeneous broadening will provide some protection against this source of dephasing, 142 because it requires the central spin to be resonant with other spins. Nevertheless, direct flip-flops likely 143 explain the shorter $T_{2}$ 's at the CTs in the $x=0.1$ sample. However, unlike the aforementioned indirect 144 spin diffusion processes, direct flip-flops can be controlled at the stage of device design through the 145 tuning/detuning of individual CT frequencies. Finally, coupling to lattice dynamics (phonons) via the CF is 
also likely to provide significant decoherence pathways, particularly as the temperature is raised. ${ }^{16}$

147 Indeed, a significant temperature dependence of $T_{2}$ is found at the CTs (more than a factor of 2 decrease

148 upon heating the sample to $7 \mathrm{~K}$ ), suggesting that $T_{2}$ may become limited by spin-lattice relaxation

$149\left(\mathrm{~T}_{1} \approx 20 \mu \mathrm{s}\right.$ at $\left.5 \mathrm{~K}\right)$. This is something that will be the subject of future investigations.

The critical result from this study is the demonstration that CTs can be employed as a means of enhancing the coherence of molecular spin qubits in concentrated samples. Therefore, instead of

153 attempting to suppress magnetic noise, which can be impractical at the stage of device design, we have 154 shown here that one can fortify the molecular spin qubit itself against this noise through the use of CTs. In 155 terms of design criteria, the molecule of choice should possess a large tunneling gap within the ground 156 magnetic doublet matching the working frequency of the EPR cavity. The key to this strategy is the 157 chemical design of molecular structures with appropriate CF states. In rare earth complexes with integer 158 spin, this goal translates into matching the $m_{J}$ components of the ground doublet with the rotational order 159 (q) of the main symmetry axis of the molecule (see Methods). While this is not trivial to achieve, the case 160 of $\mathrm{HoW}_{10}$ is not an isolated example. For example, within rigid polyoxometalate chemistry, the Terbium 161 derivative of the $\left[\mathrm{LnP}_{5} \mathrm{~W}_{30} \mathrm{O}_{110}\right]^{12-}$ series with pentagonal structure (approximate $C_{5 v}$ symmetry) has been 162 characterized as having an $m_{J}= \pm 5$ ground state with an even larger tunneling gap of $\sim 21 \mathrm{GHz}$ that may 163 be suitable for pulsed Q-band EPR. ${ }^{30}$ Tunability across this range $(10-100 \mathrm{GHz})$ is desirable and 164 practical for quantum information applications, given that it sits at the current high end of the electronics 165 spectrum. Moreover, operation at these CTs requires application of only very moderate magnetic fields 166 (<0.2 T in the present example). Of course, this strategy can and should be combined with other known 167 ideas that are already being applied with great success, such as using rigid lattices with low abundance of

168 nuclear spins. ${ }^{16}$ Nevertheless, it is remarkable that working with CTs offers the unique advantage of 169 allowing long coherence times with high concentrations of molecular spin qubits. In fact, for other 170 molecular spin qubit candidates, $T_{2}$ values of the order of tens of $\mu$ s were only observable in deuterated 171 and highly diluted samples of $\mathrm{Cr}_{7} \mathrm{Ni}$ molecular wheels ${ }^{10}$ and $\mathrm{Cu}(\mathrm{mnt})_{2}$ complexes. ${ }^{16}$

172

\section{References}

1 Schlosshauer, M. A. Decoherence: and the Quantum-To-Classical Transition. Springer (2008)

2 Stamp, P. C. E. Quantum information: Stopping the rot. Nature 453, 167-168 (2008)

2 Stamp, P. C. E. Quantum information: Stopping the rot. Nature 453, 167-168 (2008)

3 Devoret, M. H., Schoelkopf, R. J. Superconducting Circuits for Quantum Information: An Outlook. Science 339, 1169-1174 (2013)

4 Duan, L. Quantum physics: A strong hybrid couple. Nature 508, 195-196 (2014)

5 Weitenberg, C. et al. Single-spin addressing in an atomic Mott insulator. Nature 471, 319-324 (2011)

$6 \quad \mathrm{Pla}, \mathrm{J} . \mathrm{J}$. et al. A single-atom electron spin qubit in silicon. Nature 489, 541-545 (2012) 
7 Taminiau, T. H., Cramer, J., van der Sar, T., Dobrovitski, V. V., Hanson, R. Universal control and error correction in multi-qubit spin registers in diamond. Nature Nanotechnology 9, 171-176 (2014)

8 Ardavan, A. et al. Will Spin-Relaxation Times in Molecular Magnets Permit Quantum Information Processing? Phys. Rev. Lett. 98, 057201 (2007)

9 Takahashi, S. et al. Decoherence in crystals of quantum molecular magnets. Nature 476, 76-79 (2011)

10 Kaminski, D. et al. Quantum spin coherence in halogen-modified $\mathrm{Cr}_{7} \mathrm{Ni}$ molecular nanomagnets. Phys. Rev. B 90, $184419(2014)$

11 Wedge, C. J. et al. Chemical Engineering of Molecular Qubits. Phys. Rev. Lett. 108, 107204 (2012)

12 Leuenberger, M. N., Loss, D. Quantum computing in molecular magnets. Nature 410, 789-793 (2001)

13 Warner, M. et al. Potential for spin-based information processing in a thin-film molecular semiconductor. Nature 503, 504-508 (2013)

14 Graham, M. et al. Influence of electronic spin and spin-orbit coupling on decoherence in mononuclear transition metal complexes. J. Am. Chem. Soc. 136, 7623-7626 (2014)

15 Thiele, S. et al. Electrically driven nuclear spin resonance in single-molecule magnets. Science 344, 1135-1138 (2014)

16 Bader, K. et al. Room temperature quantum coherence in a potential molecular qubit. Nature Comms. 5, 5304 (2014)

17 Clemente-Juan, J. M., Coronado, E. Magnetic clusters from polyoxometalate complexes. Coord. Chem. Rev. 193-195, 361-394 (1999)

Clemente-Juan, J. M., Coronado, E., Gaita-Ariño, A. Magnetic polyoxometalates: from molecular magnetism to molecular spintronics and quantum computing. Chem. Soc. Rev. 41, 7464-7478 (2012)

19 Lehmann, L., Gaita-Ariño, A., Coronado, E., Loss, D. Spin qubits with electrically gated polyoxometalate molecules. Nature Nanotechnology 2, 312-317 (2007)

20 van Hoogdalem, K, Stepanenko, D., Loss, D. Molecular Magnets for Quantum Information Processing. J. Bartolomé et al. (eds.), Molecular Magnets, NanoScience and Technology, Springer-Verlag (2014)

21 AlDamen, M. A., Clemente-Juan, J. M., Coronado, E., Martí-Gastaldo, C., Gaita-Ariño, A. Mononuclear lanthanide single-molecule magnets based on polyoxometalates. J. Am. Chem. Soc., 130, 8874-8875 (2008)

22 Martínez-Pérez, M. J. et al. Gd-based single-ion magnets with tunable magnetic anisotropy: molecular design of spin qubits. Phys. Rev. Lett. 108, 247213 (2012)

23 AIDamen, M. A. et al. Mononuclear lanthanide single molecule magnets based on the polyoxometalates $\left[\mathrm{Ln}\left(\mathrm{W}_{5} \mathrm{O}_{18}\right)_{2}\right]^{9-}$ and $\left[\mathrm{Ln}\left(\beta-\mathrm{SiW}_{11} \mathrm{O}_{39}\right)_{2}\right]^{13-}\left(\mathrm{Ln}^{\mathrm{III}}=\mathrm{Tb}, \mathrm{Dy}, \mathrm{Ho}, \mathrm{Er}, \mathrm{Tm}\right.$, and $\left.\mathrm{Yb}\right)$. Inorg. Chem. Soc. 48, 3467-3479 (2008)

24 Ghosh, S. et al. Multi-frequency EPR studies of a mononuclear holmium single-molecule magnet based on the polyoxometalate $\left[\mathrm{Ho}^{\prime \prime \prime}\left(\mathrm{W}_{5} \mathrm{O}_{18}\right)_{2}\right]^{9-}$. Dalton Trans. 41, 13697-13704 (2012)

25 Schweiger, A., Jeschke, G. Principles of Pulse Electron Paramagnetic Resonance, Oxford University Press (2001) 

(2013)

29

30 Cardona-Serra, S. et al. Lanthanoid single-ion magnets based on polyoxometalates with a 5-fold Symmetry: The

Bollinger, J. J. et al. Laser-Cooled-Atomic Frequency Standard. Phys. Rev. Lett. 54, 1000-1003 (1985)

Vion, D. et al. Manipulating the Quantum State of an Electrical Circuit. Science 296, 886-889 (2002)

Wolfowicz, G. et al. Atomic clock transitions in silicon-based spin qubits. Nature Nanotechnology 8, 561-564

Balian, S. J, Wolfowicz, G., Morton, J. J. L., Monteiro, T. S. Quantum bath-driven decoherence of mixed spin systems. Phys. Rev. B. 89, 045403 (2014) Series $\left[\mathrm{LnP}_{5} \mathrm{~W}_{30} \mathrm{O}_{110}\right]^{12-}\left(\mathrm{Ln}^{3+}=\mathrm{Tb}, \mathrm{Dy}, \mathrm{Ho}, \mathrm{Er}, \mathrm{Tm}\right.$, and Yb). J. Am. Chem. Soc. 134, 14982-14990 (2012)

\section{Supplementary Information is linked to the online version of the paper at www.nature.com/nature.}

Acknowledgements We thank L. Song and J. van Tol for technical assistance with the X-band EPR spectrometer. This work was supported by the NSF (grant \# DMR-1309463) and the Air Force (AOARD grant \#134031). Work performed at the NHMFL is supported by the NSF (DMR-1157490) and by the State of Florida. Work performed at the ICMol is supported by the European Research Council (Grants SPINMOL and DECRESIM), by the Spanish MINECO (Projects MAT-2014-56143-R and CTQ201452758-P) and by the Generalidad Valenciana (Prometeo and ISIC-Nano Programs of excellence). AGA thanks the Spanish MINECO for a Ramón y Cajal Fellowship.

Author Contributions S.H., E.C. and A.G. conceived the research and wrote the paper. Y.D. prepared the samples. S.H., M.S. and D.K. designed the experiments, while M.S. and D.K. performed the measurements. S.H., M.S. and D.K. analyzed the results.

Author Information Reprints and permissions information is available at www.nature.com/reprints. The authors declare no competing financial interests. Correspondence and requests for materials should be addressed to S. Hill (shill@magnet.fsu.edu) or E. Coronado (eugenio.coronado@uv.es).

\section{Figure Legends}

Figure 1: HoW 10 tunneling gap. (a) Zeeman diagrams for the $m_{J}= \pm 4, I=7 / 2$ ground state, with $B_{0} / / z$ : the thin gray lines assume exact $D_{4 \mathrm{~d}}$ symmetry, while the thick black curves assume an 'axial $+B_{4}^{4} \hat{O}_{4}^{4}$ ' parameterization. $^{24}$ (Inset) The corresponding $9.64 \mathrm{GHz} \mathrm{CW} \mathrm{EPR} \mathrm{spectrum} \mathrm{(from} \mathrm{Ref.} \mathrm{[24])} \mathrm{is} \mathrm{observed}$ well below the $D_{4 \mathrm{~d}}$ prediction (gray arrow denotes expected highest field resonance), providing evidence for the tunneling gap. Indeed, the 'axial $+B_{4}^{4} \hat{O}_{4}^{4}$ ' parameterization gives excellent agreement with the data, both in terms of resonance positions (blue arrows) and intensity (arrow thickness). The red vertical lines, meanwhile, indicate the locations of CTs. (b) 3D EPR intensity map including inhomogeneous broadening 
due to a Gaussian distribution in $B_{4}^{4}\left(\sigma_{B 44}=2.1 \times 10^{-5} \mathrm{~cm}^{-1}\right)$; darker shading represents stronger intensity, with contours at the $\pm \sigma_{\Delta}$ (red) and $\pm 2 \sigma_{\Delta}$ (blue) levels $\left(\sigma_{\Delta}=123 \mathrm{MHz}\right.$, the s.d. in $\Delta$ ). Red arrows denote $\mathrm{CTs}$, dashed lines denote locations of $9.64 \mathrm{GHz}$ resonances, and the inset shows the $\mathrm{HoW}_{10}$ molecule.

Figure 2: ESE-detected spectra for a dilute sample. (a) Variable frequency measurements at $5.0 \mathrm{~K}$ for an $x=0.001$ crystal, with $\theta=29^{\circ}$; the frequencies are indicated in $\mathrm{GHz}$ above each trace. (b) Frequency versus field plot of the resonances in (a). The data are in good agreement with simulations (solid curves) based on the 'axial $+B_{4}^{4} \hat{O}_{4}^{4}$ ' parameterization [24]. Selected $\mathrm{T}_{2}$ values (in $\mu \mathrm{s}$ ) determined from the measurements in Fig. 3 are indicated close to some of the data points in (b). Vertical error bars in (b) denote pulse excitation bandwidths $\left( \pm 1 / 2 \tau_{\pi / 2}\right.$, where $\tau_{\pi / 2}$ is the duration of the $\pi / 2$ pulse), while horizontal error bars represent standard deviations ( \pm s.d.) deduced from Gaussian fits to the resonances in (a).

Figure 3: $T_{2}$ divergence at the CTs. Field-swept $T_{2}$ measurements recorded at $5.0 \mathrm{~K}$ for a small $x=$ 0.001 crystal at $\theta=22^{\circ}$ and various frequencies indicated in the right panel. The first four panels illustrate the divergences in $T_{2}$ at the CTs, referenced to the left-hand ordinate; the data are plotted in an expanded view as a function of $\left(B_{0 z}-B_{\min }\right)$, with best-fit $B_{\min }$ values given in each panel. The right-hand panel, meanwhile, displays $T_{2}$ values well away from the CTs (see Fig. 1b), referenced to the right-hand ordinate. Error bars denote the standard error in $\mathrm{T}_{2}$.

Figure 4: ESE-detected spectra for a concentrated sample. (a) Variable frequency measurements at $5.0 \mathrm{~K}$ for an $x=0.10$ crystal, with $\theta=20^{\circ}$; the frequencies are indicated in $\mathrm{GHz}$ above each trace. The ESE resonances are attributed to CTs. (b) Frequency versus field plot of the CTs in (a). Optimum $T_{2}$ values (in $\mu \mathrm{s}$ ) are indicated next to the $9.11 \mathrm{GHz}$ data. Meanwhile, the curves correspond to predictions based on the CW EPR parameterization [24]. (c) Field-swept $T_{2}$ measurements recorded at $5.0 \mathrm{~K}$ for a separate $x=0.10$ crystal at $\theta=25^{\circ}$ and frequencies of 9.12 (blue squares) and $9.20 \mathrm{GHz}$ (red circles). Vertical error bars in (b) denote pulse excitation bandwidths $\left( \pm 1 / 2 \tau_{\pi / 2}\right.$, where $\tau_{\pi / 2}$ is the duration of the $\pi / 2$ pulse), while horizontal error bars represent standard deviations ( \pm s.d.) deduced from Gaussian fits to the resonances in (a). Error bars in (c) denote the standard error in $\mathrm{T}_{2}$.

Extended Data Figure 1: $T_{2}$ scaling. Field-swept $T_{2}$ measurements for the $x=0.001$ (a) and $x=0.01$ (b) concentrations at $5 \mathrm{~K}$; the data are plotted as a function of $\left(B_{0 z}-B_{\min }\right)$ on both log-log (main panels) and linear (insets) scales. The blue lines are power-law fits to the positive $\left(B_{0 z}-B_{\min }\right)$ data (green points), with the obtained exponents given in the figures. Error bars denote the standard error in $T_{2}$.

Extended Data Figure 2: $T_{2}$ divergence at the $x=0.01$ concentration. Field-swept $T_{2}$ measurements recorded at $5.0 \mathrm{~K}$ for two separate crystals at frequencies of $9.12 \mathrm{GHz}$ (blue squares) and $9.20 \mathrm{GHz}$ (red circles). Error bars denote the standard error in $\mathrm{T}_{2}$. 
Extended Data Figure 3: ESE-detected spectra for the $x=0.01$ concentration. (a) Variable frequency measurements at $5.0 \mathrm{~K}$, with $\theta=30^{\circ}$; the frequencies are indicated above each trace. Similar to spectra for the $x=0.001$ sample, the broad $9.2 \mathrm{GHz}$ CT peak splits into two upon moving away from the tunneling gap minimum (see also Fig. 1). However, weak ESE intensity can still be detected at $B_{0 z}=165 \mathrm{mT}$ at all four frequencies. This is due to vertical broadening of the $\mathrm{CT}$, caused by a Gaussian distribution in $B_{4}^{4}$.

\section{Methods}

\section{Experimental Details}

248 Pulsed EPR measurements were performed on a commercial Bruker E680 X-band spectrometer equipped with a cylindrical $\mathrm{TE}_{011}$ dielectric resonator (model ER $4118 \mathrm{X}-\mathrm{MD} 5$ ) with a center frequency $f_{0}=9.75 \mathrm{GHz}$. Single-crystals of $\mathrm{Na}_{9}\left[\mathrm{Ho}_{x} \mathrm{Y}_{(1-x)}\left(\mathrm{W}_{5} \mathrm{O}_{18}\right)_{2}\right] \cdot n \mathrm{H}_{2} \mathrm{O}(x=0.001$ to 0.25$)$ were prepared according to the method described in Ref. [23]. Samples were re-crystallized prior to study, then transferred to the spectrometer directly from the mother liquor and cooled rapidly in order to prevent loss of crystallinity due to evaporation of lattice solvent. The sample temperature was controlled using an Oxford Instruments CF935 helium flow cryostat and ITC503 temperature controller. A strong temperature dependence of $\mathrm{T}_{2}$ at the CTs required operation of the cryostat at a base temperature of $5.0 \mathrm{~K}$ in order to ensure good thermal stability and sample-to-sample reproducibility.

For each series of measurements, a single crystal was mounted on a $4 \mathrm{~mm}$ diameter quartz rod and positioned at the center of the cylindrical resonator for perpendicular mode excitation. The tendency for samples to rapidly lose solvent, and the low symmetry $P \overline{1}$ space group of the HoW 10 compound, made it impossible to index and align crystals prior to mounting. However, the Bruker E680 and ER 4118X-MD5 dielectric resonator combination allows for in situ sample rotation about a single axis. Each crystal was therefore aligned as best as possible on the basis of angle-dependent CW EPR measurements performed at $9.75 \mathrm{GHz}$ and $5.0 \mathrm{~K}$. The remaining misalignment, $\theta$, between $B_{0}$ and $z$ was determined by scaling the applied field to match the simulations in Fig. 1 (see below). A $\theta<30^{\circ}$ criterion was then applied; crystals not meeting this condition were discarded and a new sample selected for study.

When overcoupled for ESE measurements, the bandwidth of the resonator, $\Delta f=f_{0} / Q \approx 250 \mathrm{MHz}$, where the loaded quality factor $Q \approx 40$. This is sufficient to allow variable-frequency measurements with reasonable microwave $B_{1}$ fields down to a lower limit of $\sim 9.1 \mathrm{GHz}$. The $B_{1}$ fields were independently measured under the same conditions via the Rabi oscillation frequency $\left(\Omega_{R}\right)$ of a spin- $1 / 2$ EPR standard (the organic radical bisdiphenylene-2-phenylallyl dissolved in polystyrene); $B_{1}$ values varied from $\sim 4 \mathrm{G}$ at $9.1 \mathrm{GHz}$, to $9 \mathrm{G}$ at $9.75 \mathrm{GHz}\left(\Omega_{\mathrm{R}}=11-25 \mathrm{MHz}\right.$ for $\left.s=1 / 2\right)$. A two-pulse sequence $(\mathrm{T} / 2-\tau-\mathrm{T}-\tau-$ echo, where $T$ characterizes the pulse durations and $\tau$ the delay time between pulses) was employed for all ESE measurements reported in this work. The values of $\mathrm{T}, \tau$ and the source power were optimized at 
each frequency, with the assumption that the optimum conditions correspond approximately to the Hahnecho sequence, $\pi / 2-\tau-\pi-\tau-$ echo, where $\pi$ refers to the tipping angle. For $\mathrm{T}_{2}$ measurements, $\tau$ was varied and the resultant echo amplitude then fit to a single exponential decay.

\section{Pulse Sequences}

Because the ESE measurements were performed well below the center frequency of the cavity, and due to the lack of a priori knowledge of the matrix elements associated with the observed transitions, pulse sequences were adjusted at each frequency by one of two methods: (1) the $\pi / 2$ pulse length (T/2) and source attenuation were adjusted to maximize the echo intensity relative to the spectrometer noise for the ESE-detected spectra in Figs. $2 a$ and $4 a$, thereby explaining the variability of the vertical error bars denoting excitation bandwidth (defined as $2 / \mathrm{T}$, or $1 / \tau_{\pi / 2}$, where $\tau_{\pi / 2}$ is the duration of the $\pi / 2$ pulse in the Hahn-echo sequence); and (2) Rabi oscillation measurements were used to determine the optimum $\pi / 2$ pulse length for the detailed $\mathrm{T}_{2}$ measurements displayed in Figs. 3, 4(c), and Extended Data Figs. 1 and 2 [the Rabi pulse sequence was optimized via method (1)]. On this basis, a Rabi frequency, $\Omega_{\mathrm{R}}=98 \mathrm{Mrad} \cdot \mathrm{s}^{-1}(15.6 \mathrm{MHz})$, was determined for $0 \mathrm{~dB}$ attenuation at the $\mathrm{CTs}$, resulting in a minimum $\pi / 2$ pulse length of $16 \mathrm{~ns}$ for the employed spectrometer. This corresponds to an optimum dephasing factor $Q_{\varphi}=820$, defined here as $Q_{\varphi}=\Omega_{R} \cdot T_{2}$, a figure of merit for qubit operation. We note, however, that this does not preclude shorter pulses using a more powerful microwave source, suggesting the possibility of $Q_{\varphi}$ values up to $1.5 \times 10^{6}$ using the modified definition in Ref. [9]. Interestingly, this value is identical to the one reported in Ref. [9] for an $\mathrm{Fe}_{8}$ nanomagnet, in spite of the vastly different frequencies employed in the two measurements, primarily because of the much longer coherence in the HoW 10 system. Based on knowledge of the spectrometer used for the $\mathrm{Fe}_{8}$ study, we estimate a $Q_{\varphi}=\Omega_{\mathrm{R}} \cdot \mathrm{T}_{2}$ of just 50 for $\mathrm{Fe}_{8}$; of course, the same arguments concerning limited source power apply in that case. The $\mathrm{HoW}_{10} Q_{\varphi}$ value compares favorably with other candidate molecular spin qubits using both definitions, e.g., the optimum $Q_{\varphi}\left(=\Omega_{R} \cdot T_{2}\right)$ varies from $\approx 2,000$ for the $\mathrm{Cr}_{7} \mathrm{Ni}$ wheel [7], up to $\approx 10,000$ obtained recently for a $\mathrm{Cu}^{\prime \prime}$ coordination complex [16]. However, one should bear in mind that extreme dilution/deuteration was employed in these cases.

\section{The Spin Hamiltonian}

The energy spectrum associated with the Hund's rule spin-orbit coupled ground state of the $\mathrm{Ho}^{+3}$ ion, with $L=6, S=2$, and $J=|L+S|=8$, can be described by the following effective Hamiltonian (eq. 3):

310 The double summation describes the CF interaction in terms of extended Stevens Operators $\hat{O}_{k}^{q}(k=2,4$, 3116 , and $|q| \leq k)$, with associated coefficients $B_{k}^{q}, 31,32$ and with $\hat{O}_{k}^{q}$ expressed in terms of the total electronic 
312 angular momentum operators $\hat{J}$ and $\hat{J}_{i}(i=x, y, z)$. Using this convention, the axial $(q=0)$ coefficients 313 determined from magnetic and continuous-wave (CW) EPR measurements are: ${ }^{23,24} B_{2}^{0}=0.601 \mathrm{~cm}^{-1}, B_{4}^{0}=$ $3146.96 \times 10^{-3} \mathrm{~cm}^{-1}$, and $B_{6}^{0}=-5.10 \times 10^{-5} \mathrm{~cm}^{-1}$. This parameterization results in the $m_{J}= \pm 4 \mathrm{CF}$ states lying 315 lowest in energy (Fig. 1), separated from the $m_{J}= \pm 5$ excited states by $\sim 20 \mathrm{~cm}^{-1} .{ }^{23}$ The $2^{\text {nd }}$ term in eq. 3 316 describes the hyperfine coupling between the $\mathrm{Ho}^{+3}$ electron and $I=7 / 2$ nuclear spin, resulting in the 317 observation of eight $(2 l+1)$ well-resolved electro-nuclear transitions via high-field CW EPR 318 measurements; here, $\hat{I}$ denotes the total nuclear angular momentum operator, and $\overleftrightarrow{\mathrm{A}}$ the hyperfine 319 coupling tensor, for which the parallel component, $A_{/ /}=830 \pm 10 \mathrm{MHz}$, has been determined from the high320 field CW EPR spectrum. ${ }^{24}$ The final two terms in eq. 3 respectively parameterize the electron and nuclear 321 Zeeman interactions with the local magnetic induction, $\vec{B}_{0}$, in terms of a Landé $g$-tensor $(\overleftrightarrow{g})$ and isotropic 322 nuclear $g$-factor $\left(g_{N}\right) ; \mu_{B}$ and $\mu_{N}$ represent the Bohr (electron) and nuclear magneton, respectively. The parallel component of the Landé $g$-tensor, $g_{z}=1.25(1)$, has been determined from CW EPR studies. ${ }^{22}$

In addition to the axial $(q=0)$ CF parameters, CW EPR measurements at X-band frequencies can only be accounted for by including a sizeable tetragonal $B_{4}^{4} \hat{O}_{4}^{4}\left[\hat{O}_{4}^{4}=\frac{1}{2}\left(\hat{J}_{+}^{4}+\hat{J}_{-}^{4}\right)\right]$ interaction, with $B_{4}^{4}$ $=3.14 \times 10^{-3} \mathrm{~cm}^{-1}$ (see Fig. 1a and Ref. [24] for detailed explanation). It is this term (which is allowed 328 because of a small distortion of the $\mathrm{HoW}_{10}$ molecule away from exact $D_{4 d}$ symmetry) that generates 329 avoided level crossings between $m_{J}= \pm 4$ states, as seen in Fig. 1a. In principle, the sixth order tetragonal $B_{6}^{4} \hat{O}_{6}^{4}$ interaction is also symmetry allowed. However, $\hat{O}_{6}^{4}$ contains the commutator $\left[\hat{J}_{z}^{2},\left(\hat{J}_{+}^{4}+\hat{J}_{-}^{4}\right)\right]$ and is, thus, indistinguishable from $\hat{O}_{4}^{4}$ within the truncated $m_{J}= \pm 4$ ground doublet. Therefore, we employ only 332 the $B_{4}^{4} \hat{O}_{4}^{4}$ term to capture the effects of the distortion away from exact $D_{4 d}$ symmetry. The key point is that $333 \hat{O}_{4}^{4}$ connects the $m_{J}= \pm 4$ states in $2^{\text {nd }}$-order, resulting in unusually large $(\sim 9 \mathrm{GHz})$ quantum tunneling 334 gaps. For $B_{0} / / z$, the frequencies of the resultant weakly allowed EPR transitions between these states then follow a field-dependence of the form (see Fig. 1b),

where the approximate quadratic expression applies for fields close to the gap minima, $B_{\min }$. Indeed, because $\hat{O}_{4}^{4}$ represents the only off-diagonal CF interaction in eq. 3, an almost exact mapping of the first expression of eq. 4 onto curves generated via exact diagonalization of eq. 3 is possible, yielding the following parameters: $\Delta=9.18 \mathrm{GHz}, \gamma_{z}=139.9 \mathrm{GHz} / \mathrm{T}\left(=1.25 \times 8 \times \mu_{B} / h\right.$, i.e., $\left.g_{z}=1.25\right)$, and $B_{\min }=23.6$,

$34270.9,118.1 \& 165.4 \mathrm{mT}$. This analysis assumes $B_{0} / / z$, while the experiments are typically performed with 343 a small field misalignment $(\theta \neq 0)$, as noted above. However, due to the extreme uniaxial symmetry of the $344 \mathrm{HoW}_{10}$ molecule, the perpendicular component of the effective gyromagnetic tensor associated with the $345 m_{J}= \pm 4$ doublet, $\gamma_{\perp, \text { eff }}<0.1 \mathrm{GHz} / \mathrm{T}\left(g_{\perp, \text { eff }}<0.01\right)$, resulting in a virtual insensitivity to the perpendicular 346 component of the applied field $\left(B_{0 \perp}\right)$ over the range explored in this investigation; for comparison, note 
that $\gamma_{\text {proton }} \approx 0.04 \mathrm{GHz} / \mathrm{T}$. For this reason, one can approximate the electronic Zeeman term in eq. 3 using

348 a scalar interaction of the form, $g_{z} \mu_{B} B_{0 z} \hat{J}_{z}$ (where $B_{0 z}=B_{0} \cos \theta$ ). Eq. 4 then applies quite generally at the

349 gap minima, provided the applied field is rescaled to account for any misalignment. For this reason, all

350 EPR spectra are plotted as a function of the longitudinal applied field component, $B_{0 z}$. Importantly, the derivative $d f / d B_{0 z} \rightarrow 0$ (i.e. $\gamma_{z, \text { eff }} \rightarrow 0$ ) as $B_{0 z} \rightarrow B_{\min }$, resulting in an almost complete insensitivity of the

352 EPR transition frequencies at the gap minima to magnetic noise associated with the environment, thus

353 giving rise to the strong $T_{2}$ divergences at the CTs. However, the small yet finite $\gamma_{\perp, \text { eff }}(<0.1 \mathrm{GHz} / \mathrm{T})$ likely

354 limits $T_{2}$ right at the CTs (within $\pm 0.5 \mathrm{G}$ of $B_{\min }$ ) in these studies due to the field misalignment. In fact,

$355 \gamma_{\perp, \text { eff }} \rightarrow 0$ as $B_{0} \sin \theta \rightarrow 0$, which may explain the longer $T_{2}$ values observed at the lowest field CTs in

356 Fig. 3, and also suggests that longer $\mathrm{T}_{2}$ 's may be achievable in precisely aligned samples.

\section{$\mathrm{T}_{2}$ Scaling}

359 The data displayed in Fig. 3 were obtained for a small crystal of the most dilute sample $(x=0.001)$. It is 360 the high quality of this crystal that results in the sharp $\mathrm{T}_{2}$ peaks at all four CTs (all four $B_{\min }$ locations). 361 However, it gives weak ESE signals, making it challenging to perform a detailed analysis of the scaling of

$362 \mathrm{~T}_{2}$ with $B_{0 z}$. Careful $\mathrm{T}_{2}$ measurements were therefore repeated for larger samples. Unfortunately, the 363 larger crystals are susceptible to twinning that manifests as a broadening of spectral peaks and $T_{2}$ 364 divergences, with the effect being most pronounced at the higher field CTs (see Fig. 2a). However, the 365 first CT at $B_{\min }=23.6 \mathrm{mT}$ often remains sharp (see below for explanation). Extended Data Fig. 1 displays $366 \mathrm{~T}_{2}$ measurements for the $x=0.001$ and 0.01 concentrations, plotted against $\left(B_{0 z}-B_{\min }\right)$ on both 367 logarithmic (main panels) and linear (insets) scales. Similar to the data in Fig. 3, the $T_{2}$ peaks exhibit 368 broad tails, with an apparent kink at $\left|B_{0 z}-B_{\min }\right| \approx 2 \mathrm{mT}$ for the more dilute sample. However, when 369 plotted on a log-log scale, the data follow a power-law (to within the experimental uncertainty) spanning 370 an order of magnitude in $\left(B_{0 z}-B_{\min }\right)$ for $x=0.001$, and almost two orders of magnitude for $x=0.01$, 371 particularly on the high-field sides of the $T_{2}$ peaks. This apparent monotonic behavior of the form $372 \mathrm{~T}_{2} \propto\left(B_{0 z}-B_{\mathrm{min}}\right)^{-n}$ supports our assertion that the decoherence is dominated by dipolar field fluctuations 373 that vanish as $d f / d B_{0 z} \rightarrow 0$. However, the exponent, $n$, is both sample-dependent $(n=0.33$ and 0.46 , 374 respectively, for $x=0.001$ and 0.01 ), and different from previous predictions: ${ }^{28,29} n=1$ for indirect flip-flop 375 processes (spin diffusion), and $n=2$ for instantaneous diffusion. ${ }^{25}$ We believe that sample inhomogeneity 376 is responsible for these differences in $\mathrm{HoW}_{10}$, thus masking the intrinsic $\mathrm{T}_{2}$ dependence on $B_{0 z}$, causing 377 obvious sample-to-sample variability. It is nevertheless interesting that a power-law scaling still holds, as 378 opposed, e.g., to Gaussian behavior. This clearly merits further theoretical investigation.

380 Reduced ESE intensity and faster $\mathrm{T}_{2}$ decay curves are part of the reason for the increased error bars and 381 apparent broad tails seen in Fig. 3 and Extended Data Fig. 1. In addition, ESE-Envelope-Modulation 382 (ESEEM) $^{25}$ is detectable in the decay curves recorded in these tails (not shown). However, only one to 383 two heavily damped periods of oscillation can be seen, thus adding to the error in $\mathrm{T}_{2}$ (not to mention a 
potential systematic error that is not taken into account in our analysis). It is these combined factors that likely explain the apparent kink in some of the data at $\left|B_{0 z}-B_{\min }\right| \approx 2 \mathrm{mT}$, as well as the weak variation in $T_{2}$ across the 'normal' transitions seen in the right-hand panel of Fig. 3. Interestingly, enough ESEEM periods can be detected to confirm that it is due to coupling to protons in the sample. Importantly, the ESEEM vanishes at the CTs, providing further strong evidence that the $\mathrm{Ho}^{+3}$ spin becomes decoupled from the surrounding dipolar spin bath as both $\left(B_{0 z}-B_{\min }\right)$ and $d f / d B_{0 z} \rightarrow 0$.

\section{Spectral Broadening}

392 The EPR spectra of $\mathrm{HoW}_{10}$ are inhomogeneously broadened, ${ }^{24}$ with the two main contributions originating 393 from (i) crystal twinning and (ii) strain in the off-diagonal $B_{4}^{4}$ CF parameter.

(i) Crystals of $\mathrm{HoW}_{10}$ form as long thin needles that tend to aggregate into aligned bundles. Separating single crystals from these bundles can be challenging, particularly given that removal of the samples from their mother liquor for periods of more than a few minutes leads to sample degradation. Even after separation, our measurements suggest varying degrees of mosaic spread, particularly for the larger crystals. Indeed, simulations of high-field CW EPR spectra (where the effects of the mosaicity are more pronounced than at X-band) employed a Gaussian orientational distribution with a full-width-at-halfmaximum (FWHM) of $1^{\circ}$, albeit for a small crystal; ${ }^{24}$ the distribution is considerably broader for many of the samples employed for ESE measurements. Within the context of eq. 2, this mode of disorder produces a spread in $\gamma_{z}$ and the $B_{\min }$ values, resulting in horizontal smearing of the energy levels in Fig. 1, as opposed to a vertical smearing produced by a distribution in $B_{4}^{4}$ (see below). The horizontal smearing becomes more pronounced at higher fields, akin to $g$-strain. Consequently, the EPR spectra often become broader with increasing field, as is clearly evident in Fig. 2, and less so in Fig. 4.

Although subtle, the effects of sample mosaicity are most pronounced at the CTs. The horizontal spread in the CTs results in a smearing of the divergence in $\mathrm{T}_{2}$. In general, the strongest/narrowest divergences were obtained for the smallest crystals, which have the smallest mosaic spread. It is for this reason that the data for the most dilute samples in Figs. 2 and 3 were obtained for two different crystals: the large 412 crystal employed in Fig. 2a did not produce particularly strong $\mathrm{T}_{2}$ divergences, with maximum values reaching only $\sim 2 \mu \mathrm{s}$. Meanwhile, a smaller crystal was employed in Fig. 3: this sample gave very good echoes right at the CTs, in spite of its reduced spin count; however, its ESE spectra vanish into the noise upon moving appreciably away from the CTs. These trends can be attributed both to a $T_{2}$ weighting effect, which amplifies the otherwise weak ESE signals at the CTs for the more ordered (longer $\mathrm{T}_{2}$ ) sample, and to the narrower mosaic distribution that further enhances echoes at the CTs. Multiple small samples were studied, and optimum $\mathrm{T}_{2}$ values at the CTs in the 6 to $8 \mu$ s range were found in nearly all cases for the $x=0.001$ and 0.01 samples (See Fig. 3 and Extended Data Figs. 1 and 2). 
421 (ii) Other sources of inhomogeneous broadening include: strains in the spin Hamiltonian parameters $\left(B_{k}^{q}\right.$, $422 \overleftrightarrow{\mathrm{A}}$, and $\overleftrightarrow{g}$ ), caused by microscopic disorder, and inhomogeneities in $B_{0}$ due to electron and nuclear dipolar 423 fields. The latter may be ruled out as a major source of broadening at X-band (and $5 \mathrm{~K}$ ) due to weak 424 sample magnetization and the lack of any systematic dependence of the EPR linewidth on Ho 425 concentration. Meanwhile, the only effect of the diagonal $(q=0)$ CF terms in eq. 3 is to ensure an isolated $426 m_{J}= \pm 4$ doublet ground state with $\gamma_{z}=g_{J} J \mu_{B} / h=139.9 \mathrm{GHz} / \mathrm{T}\left(J=8\right.$ and $\left.g_{J}=1.25\right)$. Other than that, the 427 low energy spectrum exhibits little or no dependence on $B_{2}^{0}, B_{4}^{0}$ and $B_{6}^{0}$, ${ }^{24}$ and should thus be insensitive to 428 strains in these parameters. For related reasons, and because of the contracted nature of the $4 f$ shell and 429 strong spin-orbit coupling, the $\overleftrightarrow{g}$ and $\overleftrightarrow{A}$ tensors are relatively immune to local strains in the crystal 430 structure (although the effective interactions will of course be sensitive to sample alignment due to the 431 strong axial character of the CF). This leaves $B_{4}^{4}$ which, indeed, has a profound influence on the X-band 432 EPR spectrum, as clearly seen in Fig. 1a and discussed in detail in Ref. [24]: $B_{4}^{4}$ directly sets the scale of 433 the tunneling gap, $\Delta$, which is responsible for the CTs.

The finite $B_{4}^{4}$ parameter arises because of a small deviation of the coordination environment around the 436 Ho ion from exact $D_{4 \mathrm{~d}}$ symmetry. ${ }^{22}$ The superposition of disorder onto this weakly distorted structure can 437 then give rise to a relatively strong modulation of the local $B_{4}^{4}$ parameter and, hence, to a broad 438 distribution for the ensemble. Working under this assumption, we re-simulated CW X-band spectra 439 obtained for an $x=0.1$ sample at a frequency of $9.64 \mathrm{GHz}$ (Fig. 8 of Ref. [24]), assuming that the main 440 source of broadening is a Gaussian distribution in $B_{4}^{4}$. The best simulation is obtained with a FWHM of $4415.0 \times 10^{-5} \mathrm{~cm}^{-1}$, i.e., $\sim 1.6 \%$ of $B_{4}^{4}$ (or a standard deviation, $\sigma_{B 44}=2.1 \times 10^{-5} \mathrm{~cm}^{-1}$ ). This, in turn, produces a 442 vertical distribution in the corresponding tunneling gap, $\Delta$. Because $\hat{O}_{4}^{4}$ connects the $m_{J}= \pm 4$ states at the $4432^{\text {nd }}$ order of perturbation, the resultant standard deviation of the gap distribution is given approximately by $444 \sigma_{\Delta} \approx 2 \Delta \sigma_{B 44} / B_{4}^{4}=4.1 \times 10^{-3} \mathrm{~cm}^{-1}=123 \mathrm{MHz}$ (FWHM of $290 \mathrm{MHz}$ ), where $\Delta=0.306 \mathrm{~cm}^{-1}=9.18 \mathrm{GHz}$ is the 445 mean gap value (the factor of '2' emerges because of the quadratic dependence of $\Delta$ on $B_{4}^{4}$ ).

447 Fig. 1b depicts the Gaussian broadening of the EPR transition frequencies as a 3D color map, with 448 contours shown at the $\pm \sigma_{\Delta}$ and $\pm 2 \sigma_{\Delta}$ levels of the distribution. Because $B_{4}^{4}$ affects only $\Delta$, this mode of 449 disorder does not shift the magnetic fields $\left(B_{\min }\right)$ at which the CTs occur for the different molecules in the 450 distribution. However, it does distribute them vertically over a relatively wide frequency range (approx. $451 \pm 0.25 \mathrm{GHz}$ at the $2 \sigma_{\Delta}$ level). This can explain the observation of ESE intensity exactly at the CTs over a 452 wide frequency range for the concentrated $(x=0.1)$ sample seen in Fig. 4 . Because the cavity employed 453 for these investigations has a center frequency at $9.75 \mathrm{GHz}$, its sensitivity improves upon increasing the 454 frequency from 9.1 to $9.4 \mathrm{GHz}$. Meanwhile, the number of $\mathrm{Ho}^{+3}$ spins in the distribution decreases with 455 increasing frequency. These two factors approximately offset, explaining the relatively constant ESE 456 intensity and signal-to-noise ratio across the studied frequency range. The ESE intensity does peak at 
9.2 GHz, above which it decays, although not as rapidly as one may expect purely on the basis of the gap distribution. This is due to the increasing $B_{1}$ field of the spectrometer, which enables excitation of more spins and hence the generation of stronger echoes at higher frequencies.

Finally, the question arises as to whether this same behavior is observable at the other concentrations. Indeed, it is. For example, CTs are very clearly observable in between the 'normal' EPR transitions over a wide frequency range at $B_{0 z}=165 \mathrm{mT}$ for the $x=0.01$ sample, as seen in Extended Data Fig. 3. Further evidence can also be found at some of the higher frequencies, where inspection of Fig. 1a reveals crossings between nuclear sub-levels $\left(\Delta m_{l}= \pm 1\right)$ at fields exactly half way between the $B_{\min }$ values. If the applied field is not well aligned to the crystal z-axis, these become avoided crossings (with $<10 \mathrm{MHz}$ gaps), giving rise to new CTs at these higher frequencies. This is a subtlety of the perpendicular field component, $B_{0 \perp}$, which will be the subject of a future publication. The avoided nuclear sub-level crossings do not influence any of the conclusions concerning the CTs at the $B_{4}^{4}$ gap minima $(\Delta)$. Nevertheless, the higher frequency CTs are observable, particularly at low fields where the effects of disorder due to sample mosaicity are less pronounced, and the 'normal' ESE transitions are quenched due to very short $\mathrm{T}_{2}$ 's. ${ }^{2,9}$ This is the explanation for the sharp double peaks seen for the $x=0.001$ sample at $\sim 50 \mathrm{mT}$ between 9.4 and $9.7 \mathrm{GHz}$ in Fig. 2a, as well as the sharp zero-field peaks and some of the fine structures seen between $B_{\min }$ values at higher fields and frequencies. On the basis of the $50 \mathrm{mTCTs}$, one can see that the vertical broadening spans less than $400 \mathrm{MHz}$ in this sample, i.e., less than $\pm 200 \mathrm{MHz}$ from the peak of the distribution. In other words, $\sigma_{B 44}$ clearly varies from sample-to-sample, being smaller for the $x=0.001$ concentration. This is the reason why intensity due to the low-frequency CTs (@ $\left.B_{\min }\right)$ is not discernible in between the broad 'normal' transitions in the most dilute sample in Fig. 2a.

\section{References}

31 Rudowicz, C., Chung, C. Y. The generalization of the extended Stevens operators to higher ranks and spins, and a systematic review of the tables of the tensor operators and their matrix elements. J. Phys.: Condens. Mat. 16, 5825-5847 (2004)

32 Stoll, S., Schweiger, A. EasySpin, a comprehensive software package for spectral simulation and analysis in EPR. J. Magn. Reson. 178, 42-55 (2006) 